\title{
Dose-dependent effects of netarsudil, a Rho-kinase inhibitor, on the distal outflow tract
}

\author{
Si Chen ${ }^{1,2,3} \cdot$ Susannah Waxman ${ }^{2} \cdot$ Chao Wang $^{2,3} \cdot$ Sarah Atta $^{2} \cdot$ Ralitsa Loewen $^{1,2} \cdot$ Nils A. Loewen $^{1,2}$ (i)
}

Received: 28 November 2019 / Revised: 24 March 2020 / Accepted: 7 April 2020 / Published online: 5 May 2020

(C) The Author(s) 2020

\begin{abstract}
Purpose To characterize the effects of netarsudil on the aqueous humor outflow tract distal to the trabecular meshwork (TM). We hypothesized that netarsudil increases outflow facility in eyes with and without circumferential ab interno trabeculectomy (AIT) that removes the TM.

Methods Sixty-four porcine anterior segment cultures were randomly assigned to groups with $(n=32)$ and without circumferential AIT $(n=32)$. Cultures were exposed to $0.1,1$, and $10 \mu \mathrm{M}$ netarsudil $(N=8$ eyes per concentration). For each concentration, IOP and vessel diameters were compared with their respective pretreatment baselines. Outflow tract vessel diameters were assessed by spectral-domain optical coherence tomography (SDOCT) and rendered in 4D (XYZ time series).

Results Netarsudil at $1 \mu \mathrm{M}$ reduced IOP both in eyes with TM $(-0.60 \pm 0.24 \mathrm{mmHg}, p=0.01)$ and in eyes without TM $(-1.79 \pm$ $0.42 \mathrm{mmHg}, p<0.01)$. At this concentration, vessels of the distal outflow tract dilated by $72 \%$. However, at $0.1 \mu \mathrm{M}$ netarsudil elevated IOP in eyes with TM $(1.59 \pm 0.36 \mathrm{mmHg}, p<0.001)$ as well as in eyes without TM $(0.23 \pm 0.32 \mathrm{mmHg}, p<0.001)$. Vessels of the distal outflow tract constricted by $31 \%$. Similarly, netarsudil at a concentration of $10 \mu \mathrm{M}$ elevated IOP both in eyes with TM $(1.91 \pm 0.193, p<0.001)$ and in eyes without TM $(3.65 \pm 0.86 \mathrm{mmHg}, p<0.001)$. At this concentration, outflow tract vessels constricted by $27 \%$.

Conclusion In the porcine anterior segment culture, the dose-dependent IOP changes caused by netarsudil matched the diameter changes of distal outflow tract vessels. Hyper- and hypotensive properties of netarsudil persisted after TM removal.
\end{abstract}

Keywords Rho-kinase inhibitor $\cdot$ Netarsudil $\cdot$ Distal outflow tract $\cdot$ Anterior chamber perfusion model $\cdot$ Porcine eyes

\section{Introduction}

Primary open-angle glaucoma (POAG) is a leading cause of irreversible blindness, with $42 \%$ of patients eventually losing vision in one eye [1]. The annual costs of glaucoma in the USA are $\$ 5.8$ billion [2]. Most POAG is treated with eye drops, but even the latest prostaglandin analogs offer continuous treatment success rates of $10 \%$ at 1 year [3]. Increased, optic nerve-damaging intraocular pressure (IOP) in POAG

Nils A. Loewen

loewen.nils@gmail.com

1 Department of Ophthalmology, University of Würzburg, Josef-Schneider-Straße 11, 97080 Würzburg, Germany

2 Department of Ophthalmology, University of Pittsburgh School of Medicine, Pittsburgh, PA, USA

3 Department of Ophthalmology, Xiangya Hospital of Central South University, Changsha, Hunan, China was long thought to be only caused by outflow resistance at the trabecular meshwork (TM), which guards the drainage system of the eye. However, data from clinical TM ablation in thousands of patients show that it fails to lower IOP to the pressure level in the recipient episcleral veins [4-8]. The data suggests that over half of resistance resides in the distal outflow tract (OT), downstream of the TM, and Schlemm's canal (SC). The loci and substrates of such distal outflow resistance are unknown, but critical to identify. New evidence of postTM outflow regulatory structures was recently presented, using automatic $3 \mathrm{D}$ segmentation and outflow reconstruction with wide-spectrum spectral-domain optical coherence tomography (SD-OCT). Outflow vessel dilation by nitric oxide correlated to a $61.5 \%$ increased outflow in porcine [9] and human eyes [10]. Recent studies of intracameral bimatoprost suggest similar effects [11].

Present ocular hypotensive medications either reduce aqueous humor production (beta-blockers, alpha-agonist, or carbonic anhydrase inhibitors) or increase the uveoscleral 
outflow as the main mechanism (prostaglandin analogs) [12]. Older muscarinic substances like pilocarpine have a direct effect on trabecular flow but fell out of favor because of their side effects that include pupillary constriction and myopization of phakic patients [13].

In this study, we examined netarsudil, a Rho-kinase inhibitor (RKI) and an inhibitor of the norepinephrine transporter [14]. It can increase the outflow facility by expanding the juxtacanalicular TM and by dilating the episcleral veins $[15$, 16]. It was approved by the US Food and Drug Administration in December 2017 as a $0.02 \%$ daily single-dose medication and is currently in phase 3 studies in Europe [17]. Contradictory observations in pilot experiments made us hypothesize that there are dose-dependent effects on the distal outflow tract that could be discovered in ex vivo porcine anterior segment cultures after removing the trabecular meshwork.

\section{Materials and methods}

\section{Study design}

Porcine eyes were obtained from a local abattoir (Thoma Meat Market, Saxonburg, Pittsburgh PA) and cultured within $2 \mathrm{~h}$ of sacrifice. No Institutional Animal Care and Use Committee approval was required because animals were not being sacrificed for the purpose of doing research. Extraocular tissues, including the conjunctiva, were carefully removed. Eyes were decontaminated by submersion in $5 \%$ povidone-iodine ophthalmic solution (Betadine 5\%, Fisher Scientific, NC9771653) for 2 min and hemisected in a biosafety cabinet. After removal of the posterior segment, lens, and ciliary body, anterior segments were mounted on custom perfusion dishes. A total of 72 eyes were cultured of which 64 eyes completed the study.

We studied the outflow facility responses of anterior segment organ cultures to $0.1,1$, and $10 \mu \mathrm{M}$ netarsudil. To establish the contribution by the TM to these responses, treatment groups were created for each concentration with and without circumferential ab interno trabeculectomy (AIT) using a trabectome as described before [18]. Eight eyes were randomly assigned to each treatment group and perfused for at least $48 \mathrm{~h}$ to establish a stable baseline IOP before treatment.

We determined the structural response of outflow tract vessels distal to the TM at the same concentrations of 1 of $0.1,1$, and $10 \mu \mathrm{M}$ netarsudil using two eyes per treatment group. Time-series volumetric scans of the perilimbal region were captured via wide-spectrum spectral-domain optical coherence tomography (SD-OCT) pre- and post-treatment. Outflow tract cross-sectional areas were compared with respective baselines.

\section{Ocular perfusion and outflow measurement}

Ocular perfusion and outflow measurements were done with a system established for the anterior chamber perfusion model $[8,18-21]$. Before each use, the system was calibrated with a water column calibration kit. Briefly, anterior segments were cultured at $37{ }^{\circ} \mathrm{C}$ and perfused at $4 \mu \mathrm{l} / \mathrm{min}$ with media (Dulbecco's modified Eagle medium (DMEM); sh30284.02, Fisher Scientific)), 1\% FBS (10082-147; Fisher Scientific), $1 \%$ antibiotic and antimycotic (15240-062; Fisher Scientific), and a microinfusion pump (70-3007; Harvard Apparatus, Holliston, MA, USA). IOP was measured at 2min intervals with pressure transducers (Deltran II: DPT200; Utah Medical Products, Midvale, UT, USA) and recorded (FE224, PL3508/P, MLA1052; ADInstruments, Sydney, Australia; LabChart 7; ADInstruments) [18]. Baseline IOP was achieved after $48 \mathrm{~h}$ of perfusion. The effect of netarsudil on IOP was observed over the subsequent time.

\section{SD-OCT imaging and analysis}

SD-OCT imaging and analysis were performed with a system established for ex vivo $[18,22,23]$ and in vivo analysis of the perilimbal sclera [24]. Each porcine eye was positioned with the optic nerve remnant secured in a low-compression mount (CryoELITE Cryogenic Vial \#W985100; Wheaton Science Products, Millville, NJ, USA) and kept damp with phosphate-buffered saline. Anterior chambers were perfused at a constant pressure of $15 \mathrm{mmHg}$ with perfusion media as done previously [18]. The eyes were placed under the sample arm of an SD-OCT equipped with a 10-mm telecentric lens (Envisu R2210, Leica, Bioptigen, Morrisville, NC, USA). The scanning beam was oriented perpendicularly to a portion of the limbus in which intrascleral signal voids of the outflow tract could be visualized. After $30 \mathrm{~min}$ of perfusion to stabilize outflow, volumetric baseline scans $(6 \times 4 \times 1.6 \mathrm{~mm})$ were captured. The medium was then supplemented with $0.1,1$, or $10 \mu \mathrm{M}$ netarsudil, and a gravity-mediated anterior chamber exchange was performed. Two eyes were imaged for each treatment group in a single session. To minimize imaging artifacts and registration errors, adjustment of the SD-OCT sample arm was limited. This permitted scans at 30-min increments in one eye for each treatment group without readjusting the sample arm. The other eye was imaged only at baseline and at the end of the 3-h experiment after careful sample arm readjustment. Each scan created 600 images, resulting in at least 5400 images analyzed per group $(0.1,1$, and $10 \mu \mathrm{M}$ netarsudil).

\section{Trabecular meshwork removal}

The TM was removed by a glaucoma surgeon experienced in $\mathrm{ab}$ interno trabeculectomy. Anterior segments were placed 
under an ophthalmic surgery microscope (S4, Carl Zeiss Meditec, Jena, Germany) and positioned with the cornea facing down in an aseptic holder. TM removal was then performed over the entire circumference via trabectome (Neomedix Corp., Tustin, California, USA) as described before [4]. TM removal was confirmed by histology.

\section{Data analysis}

IOP measurements were down-sampled into 2-h blocks and normalized to respective controls. Pretreatment was compared with post-treatment with a one-sample $t$ test in Python 3.6 [25]. SD-OCT images were processed in ImageJ [26] (version 1.50i, National Institute of Health, Bethesda, MD, USA) and Amira Aviso (version 9.1, FEI, Thermo Scientific, Waltham, MA) as done previously [18] to remove noise, to align preand post-treatment outflow tract signal voids in a threedimensional space (3D), and to allow automated, quantitative measurement of cross-sectional areas (CSA). Cross-section areas of pre- and post-treatment were compared with a student's $t$ test.

\section{Results}

Histology of eyes in which the porcine TM was left intact presented with the characteristic appearance of the aqueous angular plexus and several circumferential canal elements (Fig. 1a). The TM appeared as a prominent, multilayered structure with trabecular beams populated by TM cells, which became more condensed towards the canal elements. Histology of eyes that had passed through the experiment and in which the TM had been ablated by AIT lacked the
TM (Fig. 1b). Circumferentially running, sagittally cut, canal-like elements could be seen adjacent to the space where the TM had been removed.

Eyes with an intact TM that were exposed to $0.1 \mu \mathrm{M}$ netarsudil experienced an IOP elevation by $1.59 \pm$ $0.36 \mathrm{mmHg}(p<0.001$, Fig. 2) when compared with baseline. In contrast, eyes perfused with netarsudil at a concentration of $1 \mu \mathrm{M}$ experienced a reduction of IOP by $-0.60 \pm 0.24 \mathrm{mmHg}$ ( $80.31 \pm 48.21 \%$ reduction, $p=0.01$ ) reduction. We observed again a significant IOP elevation of $1.91 \pm 0.19 \mathrm{mmHg}$ $(p<0.001)$ at a higher concentration of $10 \mu \mathrm{M}$.

Eyes that had undergone a circumferential removal of TM by AIT showed an IOP elevation by $0.23 \pm 0.32 \mathrm{mmHg}$ at $0.1 \mu \mathrm{M}$ netarsudil $(p<0.001$, Fig. 2$)$, just like eyes with an intact TM. However, IOP was lowered by $1 \mu \mathrm{M}$ netarsudil ($1.79 \pm 0.42 \mathrm{mmHg}, p<0.001)$, as seen in nonablated eyes. At $10 \mu \mathrm{M}$, the highest concentration tested, netarsudil resulted again in an IOP elevation by $3.65 \pm 0.86 \mathrm{mmHg}(p<0.001)$, as seen in eyes with an intact TM.

SD-OCT was able to measure CSA changes of vessels of the distal outflow tract (Fig. 3). Corresponding to the IOP data, $0.1 \mu \mathrm{M}$ netarsudil caused a $50 \pm 31 \%$ reduction of the CSA of perilimbal outflow tract vessels (Fig. 4). In contrast, at $1 \mu \mathrm{M}$ netarsudil, there was a $37 \pm 14 \%$ increase in CSA due to the dilation of outflow tract vessels. At $10 \mu \mathrm{M}$ netarsudil, a constriction occurred again with a reduction of CSA by $43 \pm 7 \%$.

\section{Discussion}

Recent evidence from clinical [27-30] and laboratory [10, 18] TM ablation studies demonstrated a significant post-TM outflow resistance that might be caused by a downstream regulatory
Fig. 1 Histology of the porcine angular aqueous plexus of perfusion cultured anterior segments rotated to surgeons view. a A section from a nonablated eye shows an intact trabecular meshwork (TM) and sagittally cut, circular drainage channel segments (red arrows). b The section from an eye with circumferentially ablated TM by AIT obtained after perfusion experiments with netarsudil. The TM is removed and circumferential drainage channels are partially unroofed
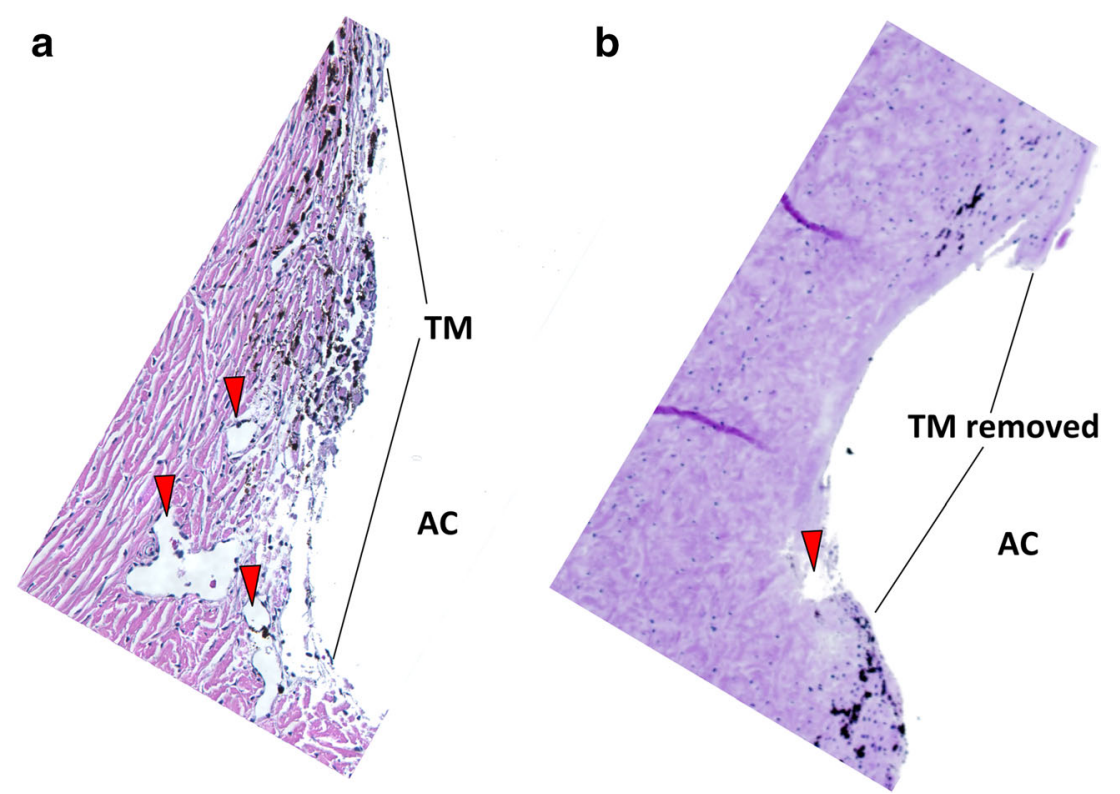
Fig. 2 Netarsudil effect on IOP in a dose-dependent manner both with and without TM $(*$ indicates significant difference from 0 , onesample $t$ test, $p<0.05$ )
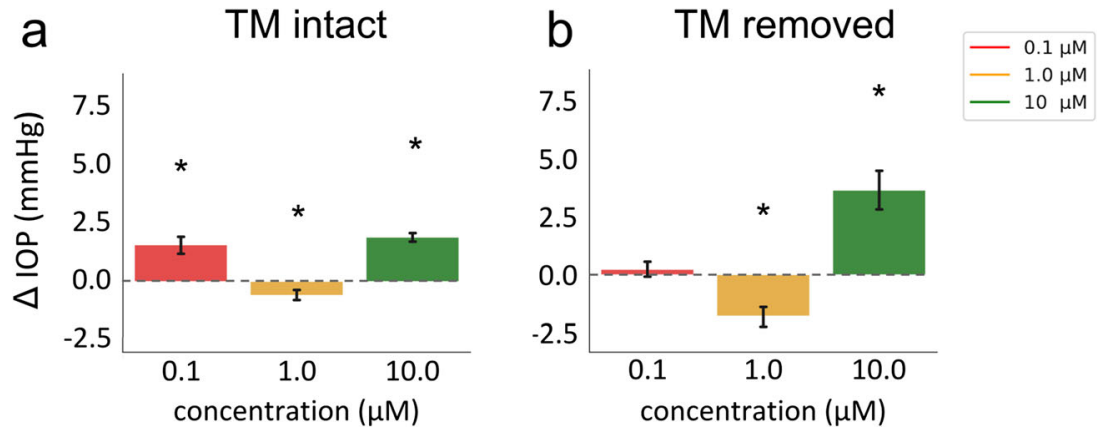

mechanism $[10,18]$. Only $0.3 \%$ of patients undergoing trabecular ablation in AIT achieve the predicted decrement in IOP to $8 \mathrm{mmHg}$ akin to the level of episcleral veins [30]. An IOP glaucoma surgery calculator we derived from those data predicts that glaucoma patients cannot achieve an IOP $<18.6 \mathrm{mmHg}$ without medications [31]. Even with topical glaucoma medications added back, TM ablation has been observed to have a failure rate of $28 \%$ within 12 months [27] for low IOP targets in moderate glaucoma, while a higher preoperative IOP is correlated to an increased postoperative IOP.

In this study, we investigated the effect of netarsudil on the structure and function of the distal outflow tract at different concentrations. Pharmacological management of post trabecular outflow resistance holds promise to patients who fail microincisional angle surgery in glaucoma because rebounding of an initially low IOP or not achieving it to start with. Interestingly, we found that while a standard concentration of $1 \mu \mathrm{M}$ of netarsudil did cause an IOP reduction and outflow vessel dilation, both a lower $(0.1 \mu \mathrm{M})$ and a higher concentration $(10 \mu \mathrm{M})$ of netarsudil had the opposite effect, resulting in IOP elevation and outflow vessel constriction. This effect did not require the TM but appeared to be mediated by distal outflow tract vessels.

The biochemistry and pharmacokinetics of netarsudil have been examined in animals $[32,33]$ and human eye models $[16$, 34] before recently entering clinical trials as a hypotensive agent for glaucoma [35, 36]. Netarsudil lowers IOP through a combination of three mechanisms, reduction of aqueous humor, increased trabecular facility, and decreased episcleral venous pressure [35]. Aqueous humor production is reduced primarily through its action on the norepinephrine transporter, while the inhibition of Rho-kinase reduces stiffness [33] and stress fibers in the TM as seen with other RKIs [21]. The impact on vessel diameters is variable, but in clinical use, conjunctival hyperemia is a common observation [37] and caused by rendering vascular smooth muscle cells less sensitive to intracellular $\mathrm{Ca}^{2+}$ [38]. Specifically, RKIs can dilate vessels by reducing myosin light chain phosphorylation of vascular smooth muscle cells [39] and by disinhibiting the endothelial nitric oxide synthase (eNOS) [40].

Our findings in porcine eyes are similar to Li et al.'s results in mouse [32] and Kiel et al.'s findings in rabbit eyes [41] who also observed dilation of outflow tract vessels with a corresponding pressure reduction. In preclinical studies, RKIs were potent inhibitors of ocular vasoconstriction [42], severe occlusive pulmonary arterial hypertension [43], and renal vasoconstriction [44] that hold potential for other chronic diseases. Release of a pathological post-trabecular outflow resistance might fit those if an outflow tract constriction and dysfunction can be confirmed in glaucoma. However, the unexpected dose-dependent IOP elevation and vasoconstriction we observed at both the lower and the higher concentration tested suggests that the opposing mechanisms are at work. The inhibition of norepinephrine reuptake is thought to increase adrenergic signaling similar to topical application of an alpha-adrenergic agonist (such as brimonidine) and to constrict ciliary body vessels, thereby reducing aqueous humor production [14]. Our experiments hint at a more narrow therapeutic window in which RKI vasodilation exceeds the effect of RKI vasoconstriction. In this regard, it is interesting to note that eyes without TM had a larger IOP increase than eyes without.
Fig. 3 Overlay of SDOCT Amira snapshots of perilimbal outflow tract structures with a progressive dilation using an example at $1 \mu \mathrm{M}$ netarsudil (red, 0 min; purple, $180 \mathrm{~min}$ ). Overlay with color other than red indicates an increased vessel diameter

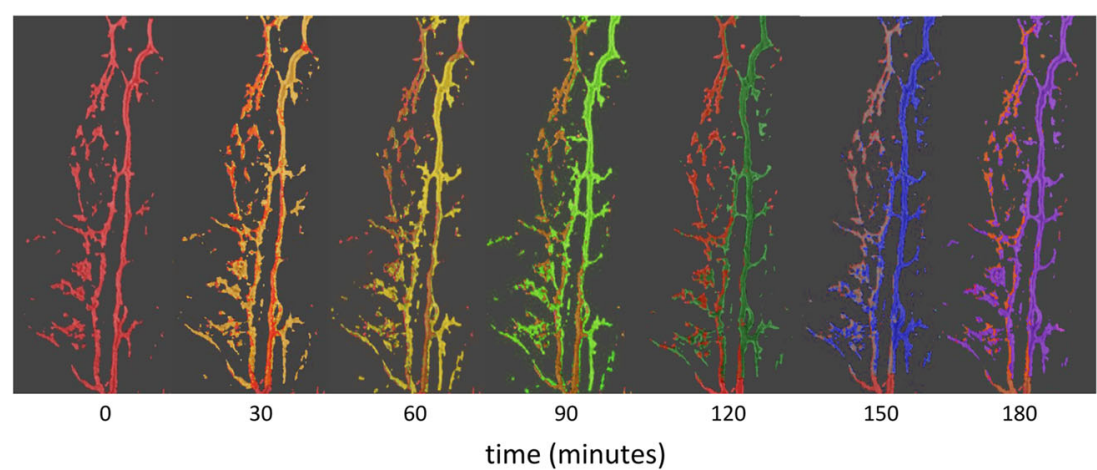



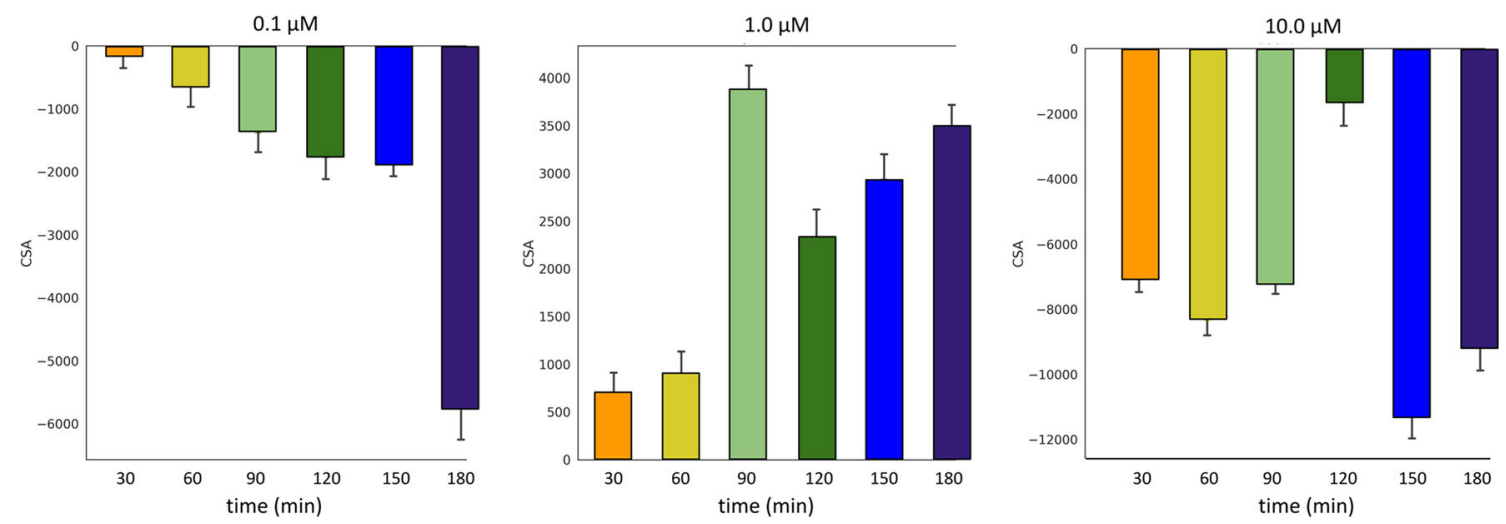

Fig. 4 Change of cross-section areas of outflow tract vessels over time at $0.1,1.0$, and $10.0 \mu \mathrm{M}$ netarsudil as measured by SDOCT (mean \pm SD)

This might be caused by the fact that TM cells express eNOS and produce nitric oxide, a potent vasodilator [45]. After TM removal, the lack of this vasodilator becomes noticeable when the balance shifts towards netarsudil-mediated vasoconstriction.

This ex vivo study had several limitations. The observed changes were statistically highly significant but small due to a perfusion rate that was only slightly higher than normal. We chose this rate out of concern to trigger $\mathrm{NO}$ release by excessive endothelial shear force [46-48]. In vivo, netarsudil might be converted more effectively into netarsudil-M1 than in this ex vivo model. Netarsudil-M1 is the esterase metabolite of netarsudil and has a greater potency [33]. Although the role of distal outflow tract resistance has been demonstrated in both porcine $[10,18]$ and human eyes [10], there might be species-dependent effect differences, especially as it relates to the extensive crosstalk between nitric oxide and RhoA/ROCK signaling [49].

In conclusion, we found that netarsudil was able not only to decrease but also increase IOP in the porcine anterior segment culture depending on the concentration tested. The IOP changes matched the diameter changes of distal outflow tract vessels. The hyper- and hypotensive property of netarsudil persisted after TM removal.

Funding information Open Access funding provided by Projekt DEAL. Unrestricted grant from the Department of Ophthalmology, University of Würzburg, Würzburg, Germany; National Eye Institute K08EY022737 (NAL); Initiative to Cure Glaucoma of the Eye and Ear Foundation of Pittsburgh (NAL); P30-EY08098 department grant (NAL); Department grant by Research to Prevent Blindness (NAL); and an unrestricted fellowship grant from the Xiangya Hospital of Central South University (SC).

\section{Compliance with ethical standards}

Conflict of interest All authors declare that they have no conflict of interest.

Ethical approval All procedures performed in studies involving human participants were in accordance with the ethical standards of the institutional and/or national research committee and with the 1964 Helsinki declaration and its later amendments or comparable ethical standards.
Statement of informed consent No approval by the Institutional Animal Care and Use Committee was required because animals were not being sacrificed for the purpose of doing research.

Open Access This article is licensed under a Creative Commons Attribution 4.0 International License, which permits use, sharing, adaptation, distribution and reproduction in any medium or format, as long as you give appropriate credit to the original author(s) and the source, provide a link to the Creative Commons licence, and indicate if changes were made. The images or other third party material in this article are included in the article's Creative Commons licence, unless indicated otherwise in a credit line to the material. If material is not included in the article's Creative Commons licence and your intended use is not permitted by statutory regulation or exceeds the permitted use, you will need to obtain permission directly from the copyright holder. To view a copy of this licence, visit http://creativecommons.org/licenses/by/4.0/.

\section{References}

1. Peters D, Bengtsson B, Heijl A (2013) Lifetime risk of blindness in open-angle glaucoma. Am J Ophthalmol 156:724-730

2. Wittenborn JS, Rein DB (2013) Cost of vision problems: the economic burden of vision loss and eye disorders in the United States. NORC at the University of Chicago, Chicago

3. Friedman DS, Quigley HA, Gelb L et al (2007) Using pharmacy claims data to study adherence to glaucoma medications: methodology and findings of the Glaucoma Adherence and Persistency Study (GAPS). Invest Ophthalmol Vis Sci 48:5052-5057

4. Dang Y, Waxman S, Wang C et al (2017) Rapid learning curve assessment in an ex vivo training system for microincisional glaucoma surgery. Sci Rep 7:1605

5. Wang C, Dang Y, Waxman S et al (2017) Angle stability and outflow in dual blade ab interno trabeculectomy with active versus passive chamber management. PLoS One 12:e0177238

6. Parikh HA, Loewen RT, Roy P et al (2016) Differential canalograms detect outflow changes from trabecular microbypass stents and ab interno trabeculectomy. Sci Rep 6:34705

7. Loewen RT, Brown EN, Scott G et al (2016) Quantification of focal outflow enhancement using differential canalograms. Invest Ophthalmol Vis Sci 57:2831-2838

8. Dang Y, Wang C, Shah P et al (2018) Outflow enhancement by three different $a b$ interno trabeculectomy procedures in a porcine anterior segment model. Graefes Arch Clin Exp Ophthalmol 256:1305-1312

9. Fallano K, Bussel I, Kagemann L et al (2017) Training strategies and outcomes of $\mathrm{ab}$ interno trabeculectomy with the trabectome. F1000Res 6:67 
10. McDonnell F, Dismuke WM, Overby DR, Stamer WD (2018) Pharmacological regulation of outflow resistance distal to Schlemm's canal. Am J Physiol Cell Physiol 315:C44-C51

11. Lee SS, Robinson MR, Weinreb RN (2019) Episcleral venous pressure and the ocular hypotensive effects of topical and intracameral prostaglandin analogs. J Glaucoma 28:846-857

12. Li T, Lindsley K, Rouse B et al (2016) Comparative effectiveness of first-line medications for primary open-angle glaucoma: a systematic review and network meta-analysis. Ophthalmology 123:129-140

13. Kass MA, Gordon M, Morley RE Jr et al (1987) Compliance with topical timolol treatment. Am J Ophthalmol 103:188-193

14. Wang R-F, Williamson JE, Kopczynski C, Serle JB (2015) Effect of $0.04 \%$ AR-13324, a ROCK, and norepinephrine transporter inhibitor, on aqueous humor dynamics in normotensive monkey eyes. $\mathrm{J}$ Glaucoma 24:51-54

15. Sturdivant JM, Royalty SM, Lin C-W et al (2016) Discovery of the ROCK inhibitor netarsudil for the treatment of open-angle glaucoma. Bioorg Med Chem Lett 26:2475-2480

16. Ren R, Li G, Le TD et al (2016) Netarsudil increases outflow facility in human eyes through multiple mechanisms. Invest Ophthalmol Vis Sci 57:6197-6209

17. Kopczynski CC, Heah T (2018) Netarsudil ophthalmic solution $0.02 \%$ for the treatment of patients with open-angle glaucoma or ocular hypertension. Drugs Today 54:467-478

18. Waxman S, Wang C, Dang Y et al (2018) Structure-function changes of the porcine distal outflow tract in response to nitric oxide. Invest Ophthalmol Vis Sci 59:4886-4895

19. Loewen RT, Roy P, Park DB et al (2016) A porcine anterior segment perfusion and transduction model with direct visualization of the trabecular meshwork. Invest Ophthalmol Vis Sci 57:1338-1344

20. Loewen N, Fautsch MP, Peretz M et al (2001) Genetic modification of human trabecular meshwork with lentiviral vectors. Hum Gene Ther 12:2109-2119

21. Dang Y, Wang C, Shah P et al (2018) RKI-1447, a Rho kinase inhibitor, causes ocular hypotension, actin stress fiber disruption, and increased phagocytosis. Graefes Arch Clin Exp Ophthalmol. https://doi.org/10.1007/s00417-018-4175-6

22. Kagemann L, Wollstein G, Ishikawa H et al (2011) 3D visualization of aqueous humor outflow structures in-situ in humans. Exp Eye Res 93:308-315

23. Kagemann L, Wang B, Wollstein G et al (2014) IOP elevation reduces Schlemm's canal cross-sectional area. Invest Ophthalmol Vis Sci 55:1805-1809

24. Nischal KK, Lathrop KL (2016) The palisades of Vogt in congenital corneal opacification (an American Ophthalmological Society thesis). Trans Am Ophthalmol Soc 114:T8

25. Virtanen P, Gommers R, Oliphant TE, et al (2020) SciPy 1.0: fundamental algorithms for scientific computing in Python. Nat Methods 17:261-272

26. Schindelin J, Arganda-Carreras I, Frise E et al (2012) Fiji: an opensource platform for biological-image analysis. Nat Methods 9:676-682

27. Bussel II, Kaplowitz K, Schuman JS et al (2015) Outcomes of ab interno trabeculectomy with the trabectome by degree of angle opening. Br J Ophthalmol 99:914-919

28. Loewen RT, Roy P, Parikh HA et al (2016) Impact of a glaucoma severity index on results of trabectome surgery: larger pressure reduction in more severe glaucoma. PLoS One 11:e0151926

29. Bussel II, Kaplowitz K, Schuman JS et al (2014) Outcomes of ab interno trabeculectomy with the trabectome after failed trabeculectomy. Br J Ophthalmol 99:258-262

30. Parikh HA, Bussel II, Schuman JS et al (2016) Coarsened exact matching of phaco-trabectome to trabectome in phakic patients: lack of additional pressure reduction from phacoemulsification. PLoS One 11:e0149384
31. Neiweem AE, Bussel II, Schuman JS et al (2016) Glaucoma surgery calculator: limited additive effect of phacoemulsification on intraocular pressure in ab interno trabeculectomy. PLoS One 11:e0153585

32. Li G, Mukherjee D, Navarro I et al (2016) Visualization of conventional outflow tissue responses to netarsudil in living mouse eyes. Eur J Pharmacol 787:20-31

33. Lin C-W, Sherman B, Moore LA et al (2018) Discovery and preclinical development of netarsudil, a novel ocular hypotensive agent for the treatment of glaucoma. J Ocul Pharmacol Ther 34:40-51

34. Wu K, Ren R, Li G et al (2017) Netarsudil increases size of giant vacuoles in Schlemm's canal of perfused human eyes. Invest Ophthalmol Vis Sci 58:1074-1074

35. Kazemi A, McLaren JW, Kopczynski CC et al (2018) The effects of netarsudil ophthalmic solution on aqueous humor dynamics in a randomized study in humans. J Ocul Pharmacol Ther 34:380-386

36. Williams RD, Novack GD, van Haarlem T et al (2011) Ocular hypotensive effect of the Rho kinase inhibitor AR-12286 in patients with glaucoma and ocular hypertension. Am J Ophthalmol 152:834-41.e1

37. Serle JB, Jay Katz L, McLaurin E et al (2018) Two phase 3 clinical trials comparing the safety and efficacy of netarsudil to timolol in patients with elevated intraocular pressure: Rho kinase elevated IOP treatment trial 1 and 2 (ROCKET-1 and ROCKET-2). Am J Ophthalmol 186:116-127

38. Uehata M, Ishizaki T, Satoh $\mathrm{H}$ et al (1997) Calcium sensitization of smooth muscle mediated by a Rho-associated protein kinase in hypertension. Nature 389:990-994

39. Amano M, Fukata Y, Kaibuchi K (2000) Regulation and functions of Rho-associated kinase. Exp Cell Res 261:44-51

40. Ming X-F, Viswambharan H, Barandier C et al (2002) Rho GTPase/ Rho kinase negatively regulates endothelial nitric oxide synthase phosphorylation through the inhibition of protein kinase B/Akt in human endothelial cells. Mol Cell Biol 22:8467-8477

41. Kiel JW, Kopczynski CC (2015) Effect of AR-13324 on episcleral venous pressure in Dutch belted rabbits. J Ocul Pharmacol Ther 31: $146-151$

42. Potts LB, Ren Y, Lu G et al (2012) Constriction of retinal arterioles to endothelin-1: requisite role of rho kinase independent of protein kinase C and L-type calcium channels. Invest Ophthalmol Vis Sci 53:2904-2912

43. Oka M, Homma N, Taraseviciene-Stewart L et al (2007) Rho kinase-mediated vasoconstriction is important in severe occlusive pulmonary arterial hypertension in rats. Circ Res 100:923-929

44. Grisk O, Schlüter T, Reimer N et al (2012) The Rho kinase inhibitor SAR407899 potently inhibits endothelin-1-induced constriction of renal resistance arteries. J Hypertens 30:980-989

45. Kim JW (2017) Comparative study of the effects of trabecular meshwork outflow drugs on the permeability and nitric oxide production in trabecular meshwork cells. Korean J Ophthalmol 31: $452-459$

46. Ashpole NE, Overby DR, Ethier CR, Stamer WD (2014) Shear stress-triggered nitric oxide release from Schlemm's canal cells. Invest Ophthalmol Vis Sci 55:8067-8076

47. Sherwood JM, Stamer WD, Overby DR (2019) A model of the oscillatory mechanical forces in the conventional outflow pathway. J R Soc Interface 16:20180652

48. Stamer WD, Braakman ST, Zhou EH et al (2015) Biomechanics of Schlemm's canal endothelium and intraocular pressure reduction. Prog Retin Eye Res 44:86-98

49. Wirth A (2010) Rho kinase and hypertension. Biochim Biophys Acta 1802:1276-1284

Publisher's note Springer Nature remains neutral with regard to jurisdictional claims in published maps and institutional affiliations. 